\title{
FACTORES ESTRESANTES DURANTE EL INTERNADO HOSPITALARIO EN INTERNOS DE ENFERMERIA- UNIVERSIDAD NACIONAL Y PRIVADAS EN UN HOSPITAL DE LA REGIÓN DE ICA SETIEMBRE 2015- AGOSTO 2016
}

\author{
Stressful factors during Internal Hospital in Nursing Institutions- National and Private Universities in a \\ Hospital of the Region of Ica September 2015- August 2016
}

Flora Martha Huisacayna Díaz ${ }^{1, a, d}$, Linda Karim Ninahuaman Huisacayna ${ }^{2, b}$ Flores ${ }^{3, a, c}$, María Elena Figueroa Cabezudo $4, a$

Yolanda Aguilar Semino de

1. Facultad de Enfermería. Universidad Nacional "San Luis Gonzaga" de Ica, Perú.

2. Facultad de Minas y Metalurgia. Universidad Nacional "San Luis Gonzaga" de Ica,Perú

3. Hospital Santa María del Socorro. Ica, Perú.

4. Hospital Augusto Hernández Mendoza. Ica, Perú.

a'Lic.en Enfermería, Ingeniero de Minas y Metalurgia, 'Especialista en Gerencia y Gestión de Servicios de Salud, 'Mag. en Educación con Mención en la Administración y Planificación de la Educación Superior.

\section{RESUMEN:}

Objetivo: Determinar los factores estresantes durante el internado hospitalario en internos de enfermería Universidad Nacional y Privadas en el Hospital Santa María del Socorro de Ica" Setiembre 2015 a Agosto 2016. Material y Métodos: Estudio cuantitativo, descriptivo transversal. Muestra constituida por 50 internos de Enfermería que realizaron internado hospitalario en el Hospital Santa María del Socorro de Ica perteneciente a Universidad Nacional y Privadas, cuestionario validado , estructurado en base del cuestionario de kezkak adaptado a las características de la muestra, con 38 ítems dividido en 4 dimensiones, Se utilizó el coeficiente Alfa-Cronbach, con un valor de 0,75. Resultados: En la dimensión : ambiente laboral, el ítem sobrecarga laboral con valor más altos $62 \%$ calificado por los internos que produce estrés demasiado; en relación al paciente: el ítem o factor estresante: encontrarse sola ante una emergencia con $60 \%$., atender a pacientes en fase terminal $72 \%$ lo calificaron como estresores que produce moderado estrés; en la dimensión socioeconómico al estresor: temer al robo a la entrada y salida del hospital por ser zona peligrosa con 44\% lo calificaron como generador de bastante estrés y la dimensión académica : el estresor :tener que ir a clase y ser evaluadas en la universidad de origen lo calificaron con $50 \%$ como estresor que produce bastante estrés. Conclusiones: Los generadores más estresantes durante el internado, se consideró: Sobrecarga laboral(asistencial y administrativo nivel 5) responsabilidad y falta de supervisión.

Palabras claves: Factores estresantes en internos de enfermería

\section{SUMMARY}

Objective: Determine stressors during the hospital internship in nursing internal - National University and private in the Santa Maria Hospital of the relief of Ica" September 2015 August 2016 methodology: study was quantitative, descriptive cross-sectional sample was formed by 50 inmates of nursing that they carried out his hospital internship at the Hospital Santa Maria of the relief of Ica" September 2015 August 2016, methodology: study was quantitative, descriptive cross-sectional sample was formed by 50 inmates of nursing that they carried out his hospital internship at the Hospital Santa Maria of the relief of Ica belonging to National University and private data collection was a direct prior consent informed the inmates, the technique was the instrument and the survey questionnaire, this was structured based on the questionnaire of kezkak adapted to the characteristics of the sample was subjected to expert opinion and the pilot for their validity and reliability the processing and analysis of the data was performed using descriptive statistics were tabulated in Excel 
table and processed.. processed by software SPSS Version 20. RESULTS: In the dimension: work environment, the work overload item with higher value $62 \%$ described by inmates that produces stress too; in relation to the patient: the item or stressor be alone to an emergency with $60 \%$,atender to patients in phase terminal $72 \%$ rated it as stressors that produces moderate stress; in the dimension socio-economic to the stressor fear to the robbery to the entry and out of hospital by be area dangerous with $44 \%$ rated it as a generator of enough stress and the ACADEMIC dimension: the stressor have to go to class and be evaluated in the university of origin lo qualified with $50 \%$ as a stressor that causes enough stress Conclusions: The most stressful generators during the internship were considered: Work overload (welfare and administrative level 5) responsibility and lack of supervision. Keywords: Stress factors in nursing interns

Key words: Stress factors in nursing interns

\section{INTRODUCCION}

Las instituciones de salud suelen ser lugares altamente estresantes para los alumnos que realizan su internado hospitalario sea cual fuera de la universidad que provienen Este escenario se observa de manera más acentuada en la carrera de enfermería, Durante el internado, el interno de enfermería se enfrenta a situaciones generadores de estrés si estos agentes generantes no son superados puede desarrollarse el estrés en sus diferentes niveles.

El estrés es el resultado el desequilibrio entre las exigencias y presiones que enfrenta el interno de enfermería, en durante el desarrollo de sus prácticas tanto del paciente, familiares del personal de salud El estrés pone a prueba la capacidad del individuo para afrontar su actividad y no sólo incluye situaciones en que la presión laboral excede la capacidad del interno El estrés laboral puede dar lugar a comportamientos disfuncionales y no habituales en el trabajo y contribuir a la mala salud física y mental del individuo Cuando el individuo está bajo presión le resulta difícil mantener un equilibrio saludable entre la vida laboral y la vida no profesional, también puede afectar al sistema inmunitario, reduciendo la capacidad del individuo para luchar contra las infecciones (1).

Las internas de enfermería al iniciar las prácticas hospitalarias, IX-X ciclo están sometidos a diferentes agentes generadores de esté como: la sobre carga laboral, la atención misma al paciente y familiares, lo académico Frente a esto, los estudiantes emplean diversos mecanismos para superar y procesar el estrés; algunos de ellos producen consecuencias negativas, como la evasión del problema, la idealización de las situaciones el ausentismo hospitalario, poca comunicación , y cambios de comportamientos desinterés, cansancio precoz, indiferencia hacia el dolor del paciente, incumplimiento de sus actividades, y roles que le corresponde, equivocaciones frecuentes, poniendo en grave problema al paciente.

El realizar el estudio fue viable porque se obtuvo información directamente de los internos de enfermería de las Universidades Nacionales y Privadas que realizan sus prácticas en el hospital Santa María del Socorro de Ica.

Los resultados de la investigación pueden servir de referencia y motivación para la réplica de estudios similares. El estudio internacional de Mamani, Omar Alejandro "Factores estresantes en las primeras experiencias prácticas hospitalarias" año 2013 en Córdova, Argentina, concluye: Los cinco factores que menos estrés ocasionan en las prácticas hospitalarias son: mala relación con los compañeros de las prácticas clínicas y rivalidad entre compañeros, todas estas en la dimensión propiamente académicas Por lo cual nos muestra que la relación entre compañero, el trabajo en conjunto no son_factores de estrés para los estudiante y que mucho menos la rivalidad. En relación a la organización de las 
prácticas la asignación de más paciente que a los demás, tener que higienizar a un paciente o tener que ayudar en la micción, y /o defecar a una persona de otro sexo no son factores de estrés para los alumnos del segundo año de enfermería que realizan las prácticas clínicas (1), otro estudio de: Izquierdo M, Rosas Cl. tesis: "Presencia de estresores en los estudiantes de enfermería en las prácticas clínicas" 2010 cuyo objetivo general fue: Identificar los estresores en los estudiantes de enfermería al realizar sus prácticas clínicas, estudio fue de tipo descriptivo ya que indagaron los estresores en estudiantes de enfermería y transversal, haciendo un corte en el tiempo, con una muestra de 98 estudiantes En este estudio, alumnos/as, manifestaron identificarse, prácticamente en todas las situaciones consideradas generadores de estrés, los principales estresores en este estudio fueron " hacer mal mi trabajo y perjudicar al paciente", “ equivocarme" y " encontrarme en una situación sin saber qué hacer", en relación a sobre carga de trabajo hay una diferencia mínima entre quienes consideran como mucho $32,7 \%$ y bastante 35,75 generadoras de estrés a esta situación (2). Giménez Tébar, J.L. "Estrés en Estudiantes de Enfermería durante sus Prácticas Clínicas en Unidad de Hospitalización a Domicilio" Por medio de este estudio dieron a conocer las situaciones que generan estrés entre las/os estudiantes de enfermería durante sus prácticas hospitalarias, en una Unidad de Hospitalización a Domicilio (UHD), y como estos factores estresantes se relacionan con variables sociodemográficas y laborales. Elaboraron un estudio transversal, descriptivo - comparativo y correlacional, con 15 estudiantes de enfermería, utilizaron el cuestionario KEZKAK modificado, como instrumento de medida. Los datos obtenidos lo analizaron estadísticamente con el programa OpenStat 11.9.08 y lo valoraron las asociaciones con un grado de significación de $p<0,05$. Como resultado obtuvieron que las/os estudiantes presentan altos niveles de estrés durante sus prácticas clínicas. Y los estresores más importantes son los relacionados con el desconocimiento, impotencia e incertidumbre que les genera una situación clínica real En el análisis destacaron que las situaciones más estresantes dentro de la práctica clínica son: "desconocimiento ante una situación clínica", "impotencia e incertidumbre ante una situación determinada" y "riesgo a dañar al paciente" (3). Un estudio nacional: Castillo Mantilla, Marie Elena. Tesis "Factores Estresantes y Desempeño de las Internas de Enfermería en las Prácticas Hospitalarias Hospital Regional Docente de Trujillo" 2013 Concluye: Que de un total de 47 internas de enfermería fue el factor estresante otros roles, el que estuvo presente en el $46,8 \%$ de ellas y el $40,4 \%$ fue el exceso de actividades. El nivel de desempeño desde la percepción de las enfermeras jefes de los servicios, en la dimensión responsabilidad fue deficiente en el 57,5\%. Para determinar la relación entre las variables del estudio se aplicó la prueba de independencia de criterios chi cuadrado, estableciéndose, como nivel de significancia estadística al $0,01(P<0,01)$. Por lo que se concluye que existe relación significativa entre los factores estresantes y el desempeño de las internas de enfermería en las prácticas hospitalarias (4).Señalándose como objetivo determinar los factores estresantes durante el internado hospitalario en Internas de Enfermería en universidades nacional y privadas en el hospital Santa María del socorro de Ica.

MATERIAL Y METODOS El estudio fue cuantitativo ,tipo descriptivo transversal La muestra estuvo conformado por 50 internos de Enfermería que realizaron su internado hospitalario en el Hospital Santa María del Socorro de Ica perteneciente a Universidad Nacional y Privadas La recolección de información fue de manera directa previo consentimiento informado a los internos, el método utilizado fue la entrevista que se realizó a los internos de enfermería la misma que fue en forma anónima e individual Se empleó la técnica de la encuesta, cuyo instrumento es el cuestionario, que consta de 
2 partes, la primera corresponde a preguntas generales y la segunda parte preguntas exclusivas a la respuesta de los objetivos Para el estudio de los estresores en estudiantes se utilizó el cuestionario bilingüe de KEZKAK modificado con algunas enunciados de sus ítems realizado por las investigadoras: contiene un total de 35 ítems los cuales fueron agrupados en 4 dimensiones: sobre el ambiente laboral, en relación al paciente, socioeconómico y el académico, cada dimensión con sus respectivas ítems: ambiente laboral con 14 ítems, en relación al paciente con 14 ítems, socio económico con 4 ítems, y académico con 3 ítems.

Los reactivos tienen opción de 6 respuestas a cada ítems en una escala de Likert: no me estreso equivalente a 0 , mínimo me estreso 1 punto, poco me estreso 2 puntos, me estreso moderado 3 puntos, me estreso bastante 4 puntos, me estreso demasiado 5 puntos .Para el análisis de resultados se agrupo por rango de acuerdo al número de ítems de cada subescala, donde a mayor valor ,mayor presencia de estresores, los valores asignados fueron $\mathbf{0 = n a d a \quad 1 =}$ minimo,2=poco,3 moderado, 4=bastante y 5= demasiado Para efectos de este estudio se ha considerado estresante aquellos ítems que obtuvieron una puntuación de igual o superior a 2 El cuestionario fue validado por 3 jueces de expertos La encuesta se aplicó faltando un mes antes de terminar su internado, quienes fueron informados sobre el propósito de la investigación, quienes dieron su consentimiento informado Asimismo en el rigor de la investigación se tuvo en cuenta la confidencialidad, credibilidad y la confortabilidad, La validez del instrumento se hizo a nivel local mediante el puntaje de 1 a 5 , con 3 expertos La validez se determinó mediante juicios de expertos se hizo uso del coeficiente y la confiabilidad, alfa de
Cronbach fue de 0,75 , la técnica de análisis e interpretación de datos fue debidamente codificada e ingresada en una base de datos utilizando el paquete estadístico SPSS Versión 14,0 para su procesamiento, luego se procedió a elaborar las tablas respectivas para atender a los objetivos trazados investigación Este trabajo se clasificó como investigación sin riesgo para los participantes los cuales fueron identificados con códigos y solo con objetivos de investigación

\section{RESULTADOS}

En la dimensión AMBIENTE LABORAL que fueron percibidos por los internos y que le causan mayor estrés son: tener poco tiempo para cumplir con el turno un $80 \%$, existencia de equipos y materiales insuficientes $y$ deteriorados el $70 \%$, sobrecarga de trabajo en un $62 \%$ realizan solas la función asistencial y administrativas el $62 \%$, trabajar con enfermeras muy exigentes y poco nada colaboradoras $66 \%$. Pincharse con agujas infectadas $68 \%$, confundirse con la medicación $66 \%$. En la dimensión AMBIENTE PACIENTE que le produce mayor estrés :atender pacientes en la fase final $72 \%$, tener que realizar procedimientos que causen dolor $70 \%$,encontrarse sola ante una situación de emergencia un $60 \%$, atender a pacientes y familiares irrespetuosos $50 \%$, atender a pacientes poco colaboradores $40 \%$,miedo a dañar al paciente $52 \%$, ver fallecer a paciente $60 \%$. En la dimensión SOCIO ECONOMICO le produce mayor estrés: no tener acceso al comedor de la institución como otras internas el $44 \%$, temer al robo hospital por ser zona peligrosa $44 \%$, tener que gastar doble pasaje $30 \%$ y no contar con material de bioseguridad por parte del hospital 30\% En la dimensión ACADEMICA: tener que ir a las clases y ser evaluadas en la universidad de origen $50 \%$, los roles no son equitativos en los servicios $40 \%$ y distanciamiento de la teoría con la práctica clínica el 30\%. 
Tabla $\mathbf{N}^{\circ} 01$ Distribución de internas de enfermería según edad

\begin{tabular}{ccc}
\hline EDAD & $\mathbf{N}^{\circ}$ & $\%$ \\
\hline $21-22 a$ & 30 & $60 \%$ \\
\hline $23-24 a$ & 19 & $38 \%$ \\
\hline $25-26 a$ & 01 & $02 \%$ \\
\hline $26+\mathbf{a}$ & -- & -- \\
\hline Total & 50 & $100.00 \%$
\end{tabular}

FUENTE: Encuestas aplicada a internos de enfermería en el hospital Santa María del Socorro de Ica de Enero- Mayo 2016

Tabla Nº2.- I DIMENSIÓN: AMBIENTE LABORAL

\begin{tabular}{|c|c|c|c|c|c|c|c|c|c|c|c|c|c|c|c|}
\hline \multirow{3}{*}{\multicolumn{2}{|c|}{$\begin{array}{l}\text { ESTRÉS } \\
\text { EACTOR } \\
\text { ESTRESANTE }\end{array}$}} & \multirow{2}{*}{\multicolumn{2}{|c|}{$\begin{array}{c}\begin{array}{c}\text { No me } \\
\text { estreso } \\
(0)\end{array} \\
0\end{array}$}} & \multirow{2}{*}{\multicolumn{2}{|c|}{$\begin{array}{c}\begin{array}{c}\text { Me estreso } \\
\text { mínimamente } \\
(1)\end{array} \\
1\end{array}$}} & \multirow{2}{*}{\multicolumn{2}{|c|}{$\begin{array}{c}\text { Me estreso } \\
\text { poco } \\
(2)\end{array}$}} & \multirow{2}{*}{\multicolumn{2}{|c|}{$\begin{array}{c}\text { Me estreso } \\
\text { moderadamente } \\
(3)\end{array}$}} & \multirow{2}{*}{\multicolumn{2}{|c|}{$\begin{array}{c}\begin{array}{c}\text { Me estreso } \\
\text { bastante } \\
(4)\end{array} \\
4\end{array}$}} & \multirow{2}{*}{\multicolumn{2}{|c|}{ 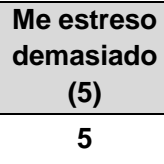 }} & \multirow{2}{*}{\multicolumn{2}{|c|}{ Total }} \\
\hline & & & & & & & & & & & & & & & \\
\hline & & & FR\% & FA & FR\% & FA & FR\% & FA & FR\% & FA & FR\% & FA & FR\% & FA & FR\% \\
\hline a) & & 01 & 02 & 02 & 04 & 02 & 04 & 06 & 12 & 08 & 16 & 31 & 62 & 50 & 100 \\
\hline b) & & 0 & 0 & 02 & 04 & 08 & 16 & 10 & 20 & 20 & 40 & 10 & 20 & 50 & 100 \\
\hline c) & & 03 & 06 & 01 & 02 & 06 & 12 & 14 & 28 & 18 & 36 & 08 & 16 & 50 & 100 \\
\hline d) & & 0 & 0 & 03 & 06 & 04 & 08 & 31 & 62 & 10 & 20 & 02 & 04 & 50 & 100 \\
\hline e) & & 05 & 10 & 16 & 32 & 23 & 46 & 03 & 06 & 02 & 04 & 01 & 02 & 50 & 100 \\
\hline f) & & 0 & 0 & 03 & 06 & 06 & 12 & 21 & 42 & 15 & 30 & 05 & 10 & 50 & 100 \\
\hline g) & & 0 & 0 & 06 & 12 & 02 & 04 & 33 & 66 & 07 & 14 & 02 & 04 & 50 & 100 \\
\hline h) & & 0 & 0 & 01 & 02 & 06 & 12 & 34 & 68 & 07 & 14 & 02 & 04 & 50 & 100 \\
\hline i) & & 0 & 0 & 02 & 04 & 11 & 22 & 29 & 58 & 04 & 08 & 04 & 08 & 50 & 100 \\
\hline j) & & 0 & 0 & 02 & 04 & 05 & 10 & 33 & 66 & 08 & 16 & 02 & 04 & 50 & 100 \\
\hline k) & & 02 & 04 & 05 & 10 & 01 & 02 & 30 & 60 & 09 & 18 & 03 & 06 & 50 & 100 \\
\hline I) & & 02 & 04 & 02 & 04 & 03 & 06 & 35 & 70 & 04 & 08 & 04 & 08 & 50 & 100 \\
\hline m) & & 02 & 04 & 01 & 02 & 15 & 30 & 25 & & 05 & 10 & 02 & 04 & 50 & 100 \\
\hline & n) & 01 & 02 & 01 & 02 & 02 & 04 & 40 & 80 & 05 & 10 & 01 & 02 & 50 & 100 \\
\hline a) & Sobreca & de t & abajo & & & & & & & & & & & & \\
\hline b) & Excesiva & spon & abilida & $y \sin s$ & rvisión & & & & & & & & & & \\
\hline c) & Ejecutar & reas & stintas & multár & nente & & & & & & & & & & \\
\hline d) & Realizar & la lo & Inción & istenc & admin & trativa & & & & & & & & & \\
\hline e) & Ausenci & e pro & colos & guías & ervicio & como & onsulta & & & & & & & & \\
\hline f) & Reducid & mbie & te de $\epsilon$ & ermer & & & & & & & & & & & \\
\hline g) & Trabajar & n enf & rmera & nuy ex & te y pc & o o na & la cola & borado & & & & & & & \\
\hline h) & Pincharn & con & guja in & tada & & & & & & & & & & & \\
\hline i) & Riesgo & ufrir $C$ & ntagio & & & & & & & & & & & & \\
\hline j) & Confund & e co & la mec & ación & & & & & & & & & & & \\
\hline k) & No ser c & az de & guarda & secr & orofesic & & & & & & & & & & \\
\hline I) & Contar c & equi & os y $\mathrm{m}$ & erial in & cientes & deter & rados & & & & & & & & \\
\hline m) & Temer a & alizar & nuevos & rocedi & ntos y $r$ & anejo & e nuev & as tecn & & & & & & & \\
\hline n) & Tener pc & tiem & o para & umplir & el turn & & & & & & & & & & \\
\hline
\end{tabular}


Tabla N 03.- II DIMENSIÓN: EN RELACIÓN AL PACIENTE

\begin{tabular}{|c|c|c|c|c|c|c|c|c|c|c|c|c|c|c|}
\hline \multirow[b]{3}{*}{$\begin{array}{l}\text { FACTOR } \\
\text { ESTRESANTE }\end{array}$} & \multicolumn{2}{|c|}{$\begin{array}{c}\text { No me estreso } \\
(0)\end{array}$} & \multicolumn{2}{|c|}{$\begin{array}{c}\text { Me estreso } \\
\text { mínimamente } \\
(1)\end{array}$} & \multicolumn{2}{|c|}{$\begin{array}{l}\text { Me estreso } \\
\text { poco (2) }\end{array}$} & \multicolumn{2}{|c|}{$\begin{array}{c}\text { Me estreso } \\
\text { moderadam } \\
\text { ente (3) }\end{array}$} & \multicolumn{2}{|c|}{$\begin{array}{c}\text { Me estreso } \\
\text { demasiado } \\
(4)\end{array}$} & \multicolumn{2}{|c|}{$\begin{array}{c}\text { Me estreso } \\
\text { demasiado } \\
\text { (5) } \\
\end{array}$} & \multirow{2}{*}{\multicolumn{2}{|c|}{ Total }} \\
\hline & \multirow[b]{2}{*}{ FA } & 0 & \multicolumn{2}{|c|}{1} & \multicolumn{2}{|r|}{2} & \multicolumn{2}{|c|}{3} & \multicolumn{2}{|r|}{4} & \multicolumn{2}{|r|}{5} & & \\
\hline & & FR \% & FA & FR \% & FA & FR \% & FA & FR \% & FA & FR \% & FA & FR \% & FA & FR \% \\
\hline a) & 03 & 06 & 03 & 06 & 05 & 10 & 17 & 34 & 20 & 40 & 02 & 04 & 50 & 100 \\
\hline b) & 02 & 04 & 02 & 04 & 12 & 24 & 20 & 40 & 11 & 22 & 03 & 06 & 50 & 100 \\
\hline c) & 03 & 06 & 05 & 10 & 03 & 06 & 36 & 72 & 02 & 04 & 01 & 02 & 50 & 100 \\
\hline d) & 02 & 04 & 01 & 02 & 02 & 04 & 25 & 50 & 17 & 34 & 03 & 06 & 50 & 100 \\
\hline e) & 01 & 02 & 05 & 10 & 14 & 28 & 18 & 36 & 11 & 22 & 01 & 02 & 50 & 100 \\
\hline f) & 02 & 04 & 03 & 06 & 04 & 08 & 30 & 60 & 10 & 20 & 01 & $02 /$ & 50 & 100 \\
\hline g) & 03 & 06 & 04 & 08 & 10 & 20 & 15 & 30 & 14 & 28 & 04 & 08 & 50 & 100 \\
\hline h) & 02 & 04 & 01 & 02 & 07 & 14 & 26 & 52 & 12 & 24 & 02 & 04 & 50 & 100 \\
\hline i) & 02 & 04 & 0 & 0 & 07 & 14 & 35 & 70 & 06 & 12 & 0 & 0 & 50 & 100 \\
\hline j) & 03 & 06 & 10 & 20 & 07 & 14 & 04 & 08 & 06 & 12 & 20 & 40 & 50 & 100 \\
\hline k) & 02 & 04 & 04 & 08 & 06 & 12 & 04 & 08 & 30 & 60 & 04 & 08 & 50 & 100 \\
\hline I) & 03 & 06 & 05 & 10 & 10 & 20 & 13 & 26 & 15 & 30 & 04 & 08 & 50 & 100 \\
\hline$\overline{\mathrm{m})}$ & 10 & 20 & 05 & 10 & 06 & 12 & 09 & 18 & 14 & 28 & 06 & 12 & 50 & 100 \\
\hline n) & 03 & 06 & 06 & 12 & 10 & 20 & 12 & 24 & 12 & 24 & 07 & 14 & 50 & 100 \\
\hline
\end{tabular}

\section{Fuente: Ibídem}
a) Atender a pacientes complejos y críticos
b) Atender a pacientes poco colaborador
c) Atender a pacientes en fase terminal
d) Atender a pacientes y familias irrespetuosas.
e) Ser afectado con el sufrimiento del paciente.
f) Encontrarme sola ante una situación de emergencia
g) Temer-a no sentirme integrada en el equipo de salud
h) Miedo a dañar al paciente
i) Realizar procedimientos que causen dolor
j) Atender a pacientes exigentes
k) Ver fallecer a paciente
l) Ser denunciado por parte de paciente y familia
m) Recibir solo críticas por personal de salud
n) Culparme de cualquier motivo (robo, infiltración y lo caída del paciente, etc.)

Fuente: Ibídem

Tabla № 04 III DIMENSIÓN: SOCIOECONÓMICO

\begin{tabular}{|c|c|c|c|c|c|c|c|c|c|c|c|c|c|c|}
\hline \multirow[t]{3}{*}{ ESTRES } & \multirow{2}{*}{\multicolumn{2}{|c|}{$\begin{array}{c}\text { No me } \\
\text { estreso } \\
(0) \\
0\end{array}$}} & \multirow{2}{*}{\multicolumn{2}{|c|}{$\begin{array}{c}\text { Me estreso } \\
\text { mínimamente } \\
(1)\end{array}$}} & \multirow{2}{*}{\multicolumn{2}{|c|}{$\begin{array}{l}\begin{array}{l}\text { Me estreso } \\
\text { poco (2) }\end{array} \\
2\end{array}$}} & \multicolumn{2}{|c|}{$\begin{array}{l}\text { Me estreso } \\
\text { loderadamen } \\
\text { te (3) }\end{array}$} & \multicolumn{2}{|c|}{$\begin{array}{c}\text { Me estreso } \\
\text { bastante } \\
(4)\end{array}$} & \multirow{2}{*}{\multicolumn{2}{|c|}{$\begin{array}{c}\begin{array}{c}\text { Me estreso } \\
\text { demasiado } \\
(5)\end{array} \\
5 \\
\end{array}$}} & \multirow{2}{*}{\multicolumn{2}{|c|}{ Total }} \\
\hline & & & & & & & & & & & & & & \\
\hline & FA & FR\% & FA & FR\% & FA & FR\% & FA & FR & FA & FR\% & FA & FR\% & FA & FR $\%$ \\
\hline a) & 02 & 04 & 07 & 14 & 10 & 20 & 15 & 30 & 10 & 20 & 06 & 12 & 50 & 100 \\
\hline b) & 01 & 02 & 03 & 06 & 04 & 08 & 10 & 20 & 22 & 44 & 10 & 20 & 50 & 100 \\
\hline \multirow{2}{*}{$\begin{array}{l}\text { c) } \\
\text { d) }\end{array}$} & 08 & 16 & 04 & 08 & 22 & 44 & 10 & 20 & 05 & 10 & 01 & 02 & 50 & 100 \\
\hline & 10 & 20 & 06 & 12 & 01 & 02 & 11 & 22 & 13 & 30 & 03 & 06 & 50 & 100 \\
\hline
\end{tabular}

a) tener que gastar doble pasaje

b) temer al robo a la entrada y salida del hospital por ser zona peligrosa

c) no tener acceso al comedor del hospital

d) no contar con material de bioseguridad por hospital

Fuente: Ibídem 
Tabla N 05. IV DIMENSIÓN: ACADÉMICA

\begin{tabular}{|c|c|c|c|c|c|c|c|c|c|c|c|c|c|c|}
\hline \multirow{3}{*}{$\begin{array}{l}\text { FACTOR } \\
\text { ESTRESANTE }\end{array}$} & \multicolumn{2}{|c|}{$\begin{array}{l}\text { No me } \\
\text { estreso } \\
(0)\end{array}$} & \multicolumn{2}{|c|}{$\begin{array}{c}\text { Me estreso } \\
\text { mínimamente } \\
\text { (1) }\end{array}$} & \multicolumn{2}{|c|}{$\begin{array}{c}\text { Me estreso } \\
\text { poco (2) }\end{array}$} & \multicolumn{2}{|c|}{$\begin{array}{l}\text { Me estreso } \\
\text { moderadam } \\
\text { ente (3) }\end{array}$} & \multicolumn{2}{|c|}{$\begin{array}{c}\text { Me estreso } \\
\text { bastante } \\
\text { (4) }\end{array}$} & \multicolumn{2}{|c|}{$\begin{array}{l}\text { Me estreso } \\
\text { demasiado } \\
\text { (5) }\end{array}$} & \multicolumn{2}{|c|}{ Total } \\
\hline & & 0 & & 1 & & 2 & & 3 & & 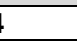 & & & & \\
\hline & FA & FR\% & FA & FR\% & FA & FR\% & FA & FR \% & FA & FR\% & FA & FR\% & FA & FR\% \\
\hline a) & 05 & 10 & 04 & 08 & 14 & 28 & 15 & 30 & 07 & 14 & 05 & 10 & 50 & 100 \\
\hline b) & 03 & 06 & 08 & 16 & 20 & 40 & 10 & 20 & 09 & 18 & 0 & 0 & 50 & 100 \\
\hline c) & 06 & 12 & 03 & 06 & 04 & 08 & 05 & 10 & 25 & 50 & 07 & 14 & 50 & 100 \\
\hline
\end{tabular}

a) Distanciamiento de la teoría con práctica clínica en el internado

b) Roles no son equitativos en los servicios

c) Tener que ir a clases y ser evaluadas en la universidad de origen

Fuente: Ibídem.

\section{Discusión}

El entorno sanitario proporciona muchos estímulos generadores de estrés, debido al contacto con las enfermedades, el dolor, pacientes en agonía, el sufrimiento del paciente y familiares, la muerte de los pacientes, atender a pacientes exigentes, asumir toda la responsabilidad del servicio (asistencial y administrativo) el hecho de tener que desempeñar un nuevo rol para el que aún no se está completamente preparado sobre todo no tener la supervisión permanente insitu por las coordinadora le produce estrés al interno De acuerdo con los resultados obtenidos en la presente investigación en relación al ambiente laboral, se observa del $100 \%$ de internos de enfermería, el $62 \%$ perciben al factor SOBRE CARGA DE TRABAJO como estresor que le produce mayor estrés en lo laboral, similares resultados se encontraron en el trabajo por Moya Nicolás, María, Larrosa Sánchez, Sandra et.al "Percepción de Estrés en los Estudiantes de Enfermería ante sus Prácticas Clínicas" donde refieren exceso sobrecarga de trabajo con una media 4,16 y con una Des. Tip. de ,98 (5), de igual manera en un trabajo de investigación de Izquierdo Lozano, Diana Mireya, Rosas Laureano, Clara Bella (2) , "PRESENCIA DE ESTRESORES EN LOS ESTUDIANTES DE ENFERMERIA EN LAS PRACTICAS CLINICAS" sobrecarga consideran mucho $32,7 \%$ y $35,75 \%$ como generadores de estrés (2), otro trabajo de investigación con resultados similares de resultados Giménez Tébar, J.L. "Estrés en estudiantes de enfermería durante sus Prácticas clínicas en unidad de hospitalización a domicilio" refiere sobre exceso de sobrecarga de trabajo 2,48 como media y desviación 0,84 (3).

Estos datos también se contrastan con la tesis realizada por Omar Alejandro Mamani quien refiere con 4,6 de media considera como factor estresante Sobre riesgo a sufrir contagio se encontró que del $100 \%$ de internos de enfermería el $58 \%$ de internos consideran al factor riesgo a sufrir contagio como estresor moderado (1), otro estudio que difiere: tesis de Mamani CCama, Danitza Deisy Tesis "factores estresantes en la primeras experiencias Prácticas hospitalarias, en estudiantes del quinto semestre de la facultad de enfermería, Universidad Nacional del Altiplano Puno 2014".en sus resultados reflejan que del $100 \%$ de internos perciben miedo a contagiarse en un $35,8 \%$ como algo factor estresante (6). Hallazgos similares se encontró en un revista de enfermería de Collados Sánchez, Juana María, García Cutillas, Nuria "Riesgo de estrés en Estudiantes durante las Prácticas Clínicas" Murcia España. RECIEN Revista Científica de Enfermería 2012, del 100\% de estudiantes masculinos el $42,85 \%$ considera como factor estresante bastante a miedo a contagiarse, en el mismo trabajo , las mujeres en un $52,17 \%$ como factor estresante de mucho (7). 
Otro trabajo que hace referencia al miedo de contagiarse es de Moya Nicolás, María, Llarosa Sánchez, Sandra et.al "Percepción de Estrés en los Estudiantes de Enfermería ante sus Prácticas Clínicas" Revista electrónica trimestral de enfermería $N^{\circ} 31$ Enfermería Global AdministraciónGestión-Calidad n' 31- Refieren 4,27 como media y 1,12 de desviación valores altos y similares al a los internos que hacen referencia durante sus prácticas pre profesionales en el hospital María de Socorro de Ica Confrontando con resultados de Omar Alejandro Mamani (1), donde refiere un 3,63 de media y como factor estresante

\section{Correspondencia:}

Mag. Flora Martha Huisacayna Díaz

Correo electrónico: martha.f.h.d@gmail.com

\section{REFERENCIAS BIBLIOGRAFICAS}

1. Mamani A. "Factores Estresantes en las Primeras Experiencias Prácticas Hospitalarias" Informe de Investigación Universidad Nacional de Córdova facultad de Ciencias Médicas Escuela de enfermería Córdova, Argentina; 2013 p. 5, $6,11,12$

2. Izquierdo D, Rosas CL. Presencia de Estresores en los Estudiantes de Enfermería en las Prácticas Clínicas Tesis Pre-grado. Veracruz, México. Facultad de Enfermería, Universidad Veracruzana: universidad docente Multidisciplinaria de Ciencias de la Salud y Trabajo Social; 2010. p .24 al 31

3. Giménez Tébar, J.L. Estrés en Estudiantes de Enfermería durante sus Prácticas Clínicas en Unidad de Hospitalización a Domicilio. Tesis docente.
Alicante, España. Departamento de Enfermería, Facultad de Ciencias de la Salud. Universidad de Alicante y Hospital General Universitario de Alicante.

4. Castillo M. Factores Estresantes y Desempeño de las Internas de Enfermería en las Prácticas Hospitalarias Hospital Regional Docente de Trujillo. Tesis Pregrado. Trujillo, Perú. Facultad de Ciencias de la Salud, Escuela Profesional de Enfermería, Universidad Privada Antenor Orrego; 2013

5. Moya M, Larrosa S. et.al. Percepción de Estrés en los Estudiantes de Enfermería ante sus Prácticas Clínicas. Murcia, España. Rev. Enfermería Global Administración- Gestión-Calidad. 2013; 12(31): 236, 237238

6. Mamani D. Factores Estresantes en la Primeras Experiencias Prácticas Hospitalarias, en Estudiantes del Quinto Semestre de la Facultad de Enfermería, Universidad Nacional del Altiplano Puno 2014. Tesis Pregrado. Puno, Perú. Facultad de Enfermería, Universidad Nacional del Altiplano; 2014. p. 59-62

7. Collados J, García N. Riesgo de Estrés en Estudiantes durante las Prácticas Clínicas Murcia, España. RECIÉN Rev. Científica de Enfermería. 2012 (4) : p. 6,7, 10

8. Meza E, Pinedo V, Sinti S. "Estresores y estrés percibido en las prácticas clínicas, en estudiantes de enfermería, de la universidad nacional de la amazonia peruana lquitos, 2014" tesis pregrado.Iquitos, Perú 2014 pg. 33,34

Recibido: 16/09/16

Aprobado para Publicación: 20/05/2017 